\title{
Intrinsic transport properties of nanoporous graphene highly suitable for complementary field-effect transistors
}

\author{
Jing Li \\ Univ. Grenoble Alpes, CNRS, Institut NÉEL, F-38042, Grenoble, France \\ E-mail: jing.li.phy@gmail.com \\ Christophe Delerue \\ ULille, CNRS, Centrale Lille, ISEN, UPHF, UMR 8520 - IEMN, F-59000 Lille, France \\ E-mail: christophe.delerue@iemn.univ-lille1.fr \\ January 2019
}

\begin{abstract}
The recent success to synthesize an ordered array of pores in graphene by a bottom-up approach [C. Moreno et al. 2018 Science 360, 199] yields a semiconducting nanoporous graphene with a bandgap of $0.6 \mathrm{eV}$. In this paper, we present calculations of the intrinsic carrier mobility in this new type of two-dimensional material. Using a fully atomistic approach, we show that carriers are mostly scattered by acoustic phonons, approximately like in semiconducting carbon nanotubes. The carrier mobility shows strong anisotropy and is as high as $800 \mathrm{~cm}^{2} /(\mathrm{Vs})$ at low carrier density. Such a high mobility, together with symmetric properties of electrons and holes, suggests that porous graphene is a promising candidate for next generations of complementary field-effect transistor technology.
\end{abstract}

Keywords: graphene, nanoporous graphene, mobility, phonon scattering

Submitted to: 2D Mater.

\section{Introduction}

As scaling of Complementary Metal Oxide Semiconductor (CMOS) technologies reaches its limits, two-dimensional (2D) materials cleaved from layered solids (hereafter, 2D materials) represent interesting alternatives to thin silicon layers for beyond-CMOS technologies $[1,2,3,4,5,6]$. Among many reasons, 2D materials are very attractive for their absence of surface dangling bonds, for their ultimate thickness which favors the electrostatic control of field-effect transistor channels, and for their possible hybridization with other 2D materials or more conventional semiconductors enabling 
the fabrication of original devices based on horizontal or vertical transport. Graphene was the first prototype of 2D materials [7]. In spite of its remarkable physical properties, it is not suitable for beyond-CMOS digital technologies due to its gap-less electronic structure. A lateral quantum confinement can be used to open a bandgap in Graphene Nano-Ribbons (GNRs) but a ribbon width below $\sim 6 \mathrm{~nm}$ is required to have a significant bandgap $(>0.2 \mathrm{eV})[8,9,10,11,12]$. Moreover, the electronic properties of GNRs depend on their edge geometry which is challenging to control.

Beyond graphene, a wide variety of 2D materials have recently received considerable attention such as Transition Metal Dichalcogenides (TMDs) [1, 13, 14, 15, 16, 17, 18, $19,20,21,22]$ or black phosphorus [23, 24, 25, 26, 27, 28]. Interestingly, record values above $100 \mathrm{~cm}^{2} /(\mathrm{Vs})$ have been reported for the room-temperature carrier mobility in monolayers of these materials, [14, 16, 28, 20] and larger ones are found in multilayers. These values can be compared to the mobility of $250 \mathrm{~cm}^{2} /(\mathrm{Vs})$ found in 2-nm-thick silicon films [29]. However, by comparison with calculated phonon-limited mobilities $[30,31,32,33,34,35]$, even accounting for their significant dispersion, one can conclude that the electrical properties of these ultra-thin 2D materials are still limited by extrinsic factors, i.e., the interaction with the environment, surface adsorption and point defects.

The family of 2D materials encompasses many other compounds [36], giving us the possibility to determine the best candidates for the development of Complementary Field-Effect Transistor (CFET) technologies. In the following, we consider a new family of 2D materials, nanoporous graphene (NPG) in which a periodic array of nanosize pores turns the semimetallic graphene into a semiconductor [37, 38]. Remarkably, NPG was recently synthesized using a bottom-up approach [39, 40, 41]. The self-assembling of molecular precursors allows to define $2 \mathrm{D}$ carbon materials at atomic precision, with geometrical patterns as small as 1 nanometer in size [42, 39, 40, 41].

CFET technologies based on NPG could present several advantages. First, NPG has a finite bandgap, semiconducting properties, and an intrinsic electron-hole symmetry. Second, NPG could be encapsulated between two insulating BN layers. This approach applied to graphene allows to obtain room-temperature carrier mobilities near the theoretical phonon-limited one [43]. Third, technological processes developed for semiconducting carbon nanotubes to make perfectly-symmetric CFETs [44, 45] could be easily transferred to NPG. Perfect ohmic contacts could be made either to the valence band using $\mathrm{Pd}$ [46] or to the conductance band using Sc [47]. In addition to these important features, we predict in this paper that NPG samples synthesized in Ref. [39] should be characterized by a high intrinsic (phonon-limited) carrier mobility, up to 800 $\mathrm{cm}^{2} /(\mathrm{Vs})$. This prediction is made using atomistic calculations in which we consider all numerous phonon scattering channels which are allowed due to the large number (240) of carbon atoms per unit cell. We present calculations of the carrier mobility not only in NPG but also in specific GNRs which are intermediate building blocks that appear during the synthesis [39]. 

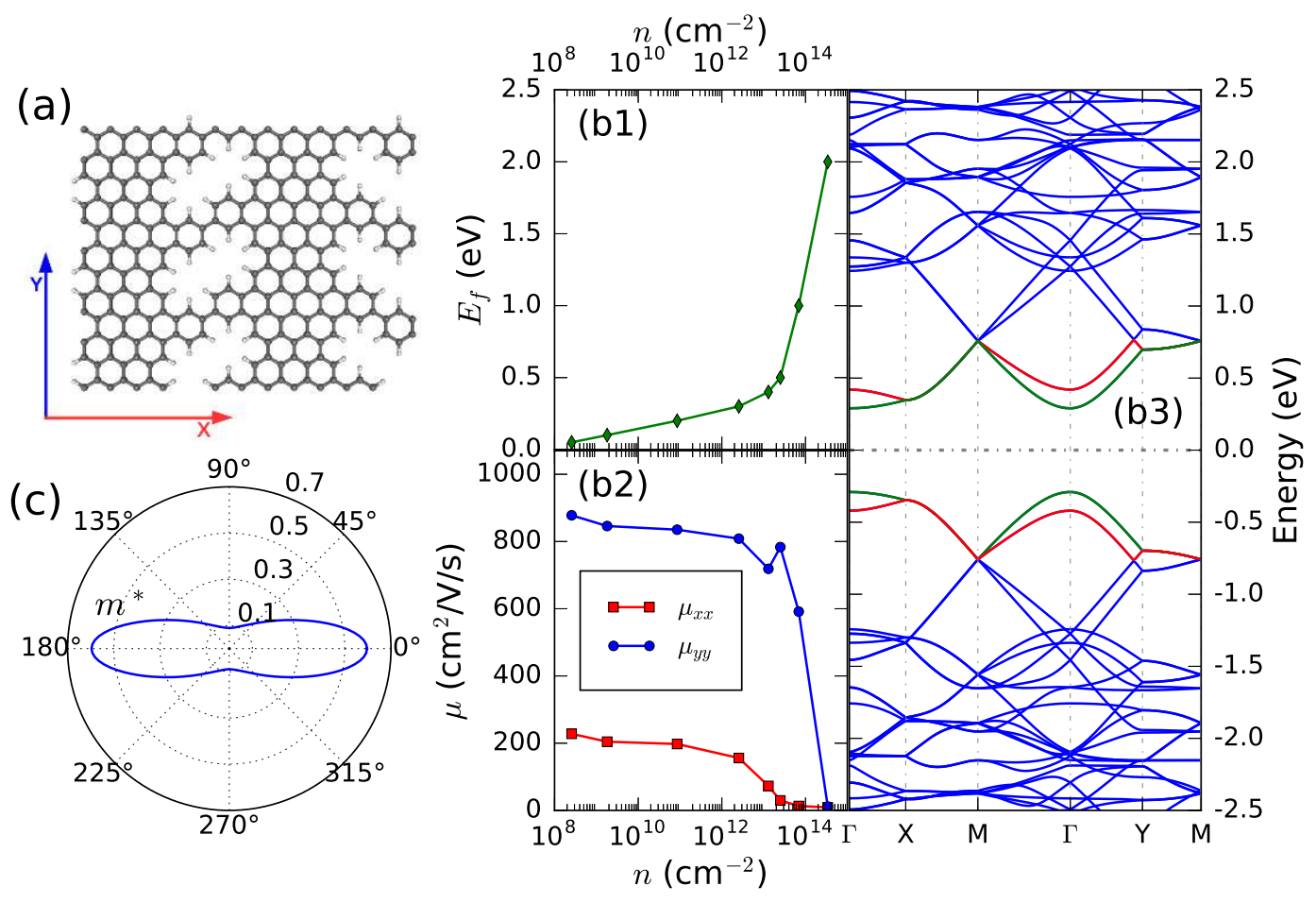

Figure 1. (a) The unit cell of the NPG. The Y axis is along the 7-13-AGNRs, the $\mathrm{X}$ axis is perpendicular. (b1) Position of the Fermi level with respect to the center of the bandgap, and (b2) the intrinsic electron mobility along the two principle axes (X axis: red line with squares; Y axis: blue line with dots), versus electron density. (b3) The electronic structure along high symmetry lines. The upper part of (b3) is aligned with the energy in (b1), in order to relate positions of the Fermi level in the electronic structure with the electron densities and the mobilities in (b2). The bands close to the energy gap are shown with different colours for clarity. (c) The anisotropic effective mass in the lowest conduction band as a function of the angle with respect to the $\mathrm{X}$ axis.

\section{Method}

The intrinsic mobility of electrons (symmetrically, holes) is computed using a fully atomistic approach, which is formally the same as in our previous studies on GNRs [48], carbon nanotubes, graphene [49] and other semiconducting structures [50]. In short, atomistic tight-binding is employed to compute the electronic structure, an atomistic force-constant model is used for phonon dispersion. The tight-binding parameters and the parameters in the force constant model are the same as in Ref. [49]. The lowfield carrier mobility is determined by solving the Boltzmann transport equation, in which the scattering terms include all possible electron-phonon couplings. For graphene, this approach gives mobilities versus carrier density and temperature [49] in excellent agreement with first-principles calculations [51, 52] and with experiments [53, 54].

The unit cell of the NPG sample that we have investigated is presented in figure 1(a). As described in Ref. [39], three reaction steps are necessary to form the $2 \mathrm{D}$ material. 

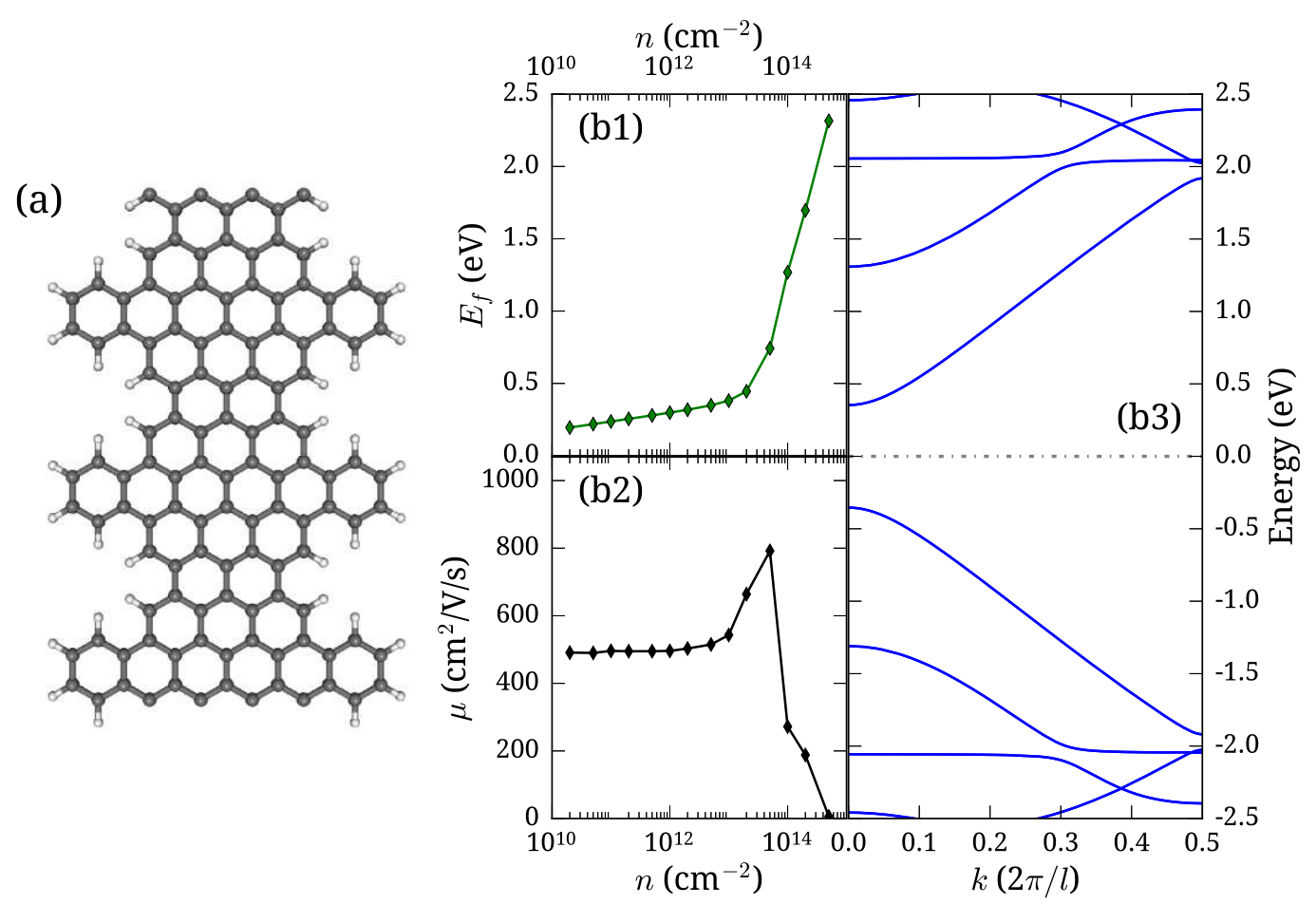

Figure 2. (a) The unit cell of the 7-13-AGNRs. (b1) Position of the Fermi level with respect to the center of the bandgap, and (b2) the intrinsic electron mobility, versus electron density. (b3) The electronic structure. The upper part of (b3) is aligned with the energy in (b1), in order to relate positions of the Fermi level in the electronic structure with the electron densities and the mobilities in (b2).

The first two steps are used to synthesize 7-13-Armchair GNRs (7-13-AGNRs), shown in figure 2(a), which are GNRs characterized by particular armchair edge structures forming consecutive pairs of 7 and 13 carbon atoms. The final reaction step interconnects 7-13-AGNRs laterally by means of a highly selective cross-coupling (figure 1(a)).

A main challenge in these calculations came from the large unit cell of NPG $\left(3 \times 2.4 \mathrm{~nm}^{2}\right)$ containing 240 carbon atoms. All possible scattering events are considered and linearized Boltzmann transport equation is solved exactly [49]. Brillouin zone integrations are performed on a non-homogeneous 1D grid for 7-13-AGNRs and on an triangular mesh for NPG (e.g., $1218 k$-points in 1D and 3394 triangles in 2D for a surface carrier density of $2.5 \times 10^{12} \mathrm{~cm}^{-2}$ and $\left.T=300 \mathrm{~K}\right)$. It was also important to validate the numerical method. In the case of pristine graphene, we checked that identical mobilities are obtained using the unit cell (2 atoms) and the large $3 \times 2.4 \mathrm{~nm}^{2}$ supercell (312 atoms).

\section{Results}

The band structures calculated for NPG and for the 7-13-AGNR are presented in figure 1(b3) and figure 2(b3), respectively. The 7-13-AGNR is characterized by a 
bandgap of $0.71 \mathrm{eV}$, which is $0.13 \mathrm{eV}$ larger than the $0.58 \mathrm{eV}$ bandgap of NPG. Such findings are fully consistent with the DFT study of Ref. [39], which gives gap values of $0.74 \mathrm{eV}$ for the $7-13-\mathrm{AGNR}$, and $0.62 \mathrm{eV}$ for $\mathrm{NPG}$. The small $(\approx 0.1 \mathrm{eV})$ difference between 7-13-AGNR and NPG bandgaps is consistent with the weak band dispersion of the NPG band structure along the $\mathrm{X}$-axis corresponding to the direction perpendicular to the 7-13-AGNR. This also explains the large effective mass in the $\mathrm{X}$ direction (figure 1(c)). Interestingly, due to this strong anisotropy in the band structure, it was recently predicted that electron waves injected from the tip of a scanning tunneling microscope should behave similarly to photons in coupled waveguides [55].

Figure 1(b2) presents the room-temperature intrinsic electron mobility as a function of the electron density $n$ along the two principle axes indicated in figure 1(a). At low electron densities $\left(n<10^{12} \mathrm{~cm}^{-2}\right)$, the mobility along the $\mathrm{Y}$-axis is about $800 \mathrm{~cm}^{2} /(\mathrm{V} \cdot \mathrm{s})$, and merely $200 \mathrm{~cm}^{2} /(\mathrm{V} \cdot \mathrm{s})$ along the X-axis. Such a strong direction-dependent mobility is consistent with the electronic structure presented by figure 1(b3). The curvature of the lowest conduction band along $\Gamma-Y$ is obviously much larger than that along $\Gamma-X$, indicating a lighter effective mass along the $\mathrm{Y}$-axis. Indeed, the effective mass along $\mathrm{Y}$ is about $0.09 m_{e}$ only (figure 1(c)), comparable to $0.085 m_{e}$ the effective mass of the 713-AGNR, whereas it is roughly $0.59 m_{e}$ along $\mathrm{X}$, about 6 times larger. The anisotropy in the effective mass explains primarily the factor 4 on the mobility between the two directions.

The mobility decreases dramatically when the electron density is increased above a certain threshold. This happens when the Fermi level lies above the conduction band minimum and the contribution from the second subband becomes important. It is noticeable that the mobility starts to drop at lower density along $\mathrm{X}\left(n \sim 10^{12} \mathrm{~cm}^{-2}\right)$ than along $\mathrm{Y}\left(n \sim 10^{13} \mathrm{~cm}^{-2}\right)$. The second subband has opposite curvature along $\Gamma-\mathrm{X}$ and $\Gamma$-Y direction, resulting in different contributions to the electron mobility. It is also worth pointing out the local minimum of electron mobility along $\mathrm{Y}$ at about $n=10^{13}$ $\mathrm{cm}^{-2}$, where the Fermi level lies between the two lowest subbands, and electron suffers from phonon scatterings to both subbands.

To further reveal the transport properties of NPG, a comparative investigation with its building block, the 7-13-AGNR, was carried out. Figure 2(b2) shows that the electron mobility in the 7-13-AGNR at low carrier density is about $500 \mathrm{~cm}^{2} /(\mathrm{Vs})$, which is smaller but comparable to the mobility of NPG along the $\mathrm{Y}$ direction $\left[800 \mathrm{~cm}^{2} /(\mathrm{Vs})\right]$. The reduction of $300 \mathrm{~cm}^{2} /(\mathrm{Vs})$ indicates that electron-phonon coupling is enhanced by the lateral confinement, an effect which is commonly found in semiconductor nanostructures [56]. At high electron density, the electron mobility first increases and then decreases. The increase of the mobility is explained by the suppression of phonon scattering to the bottom of the lowest subband as a consequence of the shift of the transport energy window. This phenomenon holds until the second subband falls inside the transport energy window, which enables inter-subband phonon scattering.

Figure 3(a) shows that, in NPG, the electrons are mostly scattered by acoustic phonons, as already found in semiconducting carbon nanotubes [49] which are also 

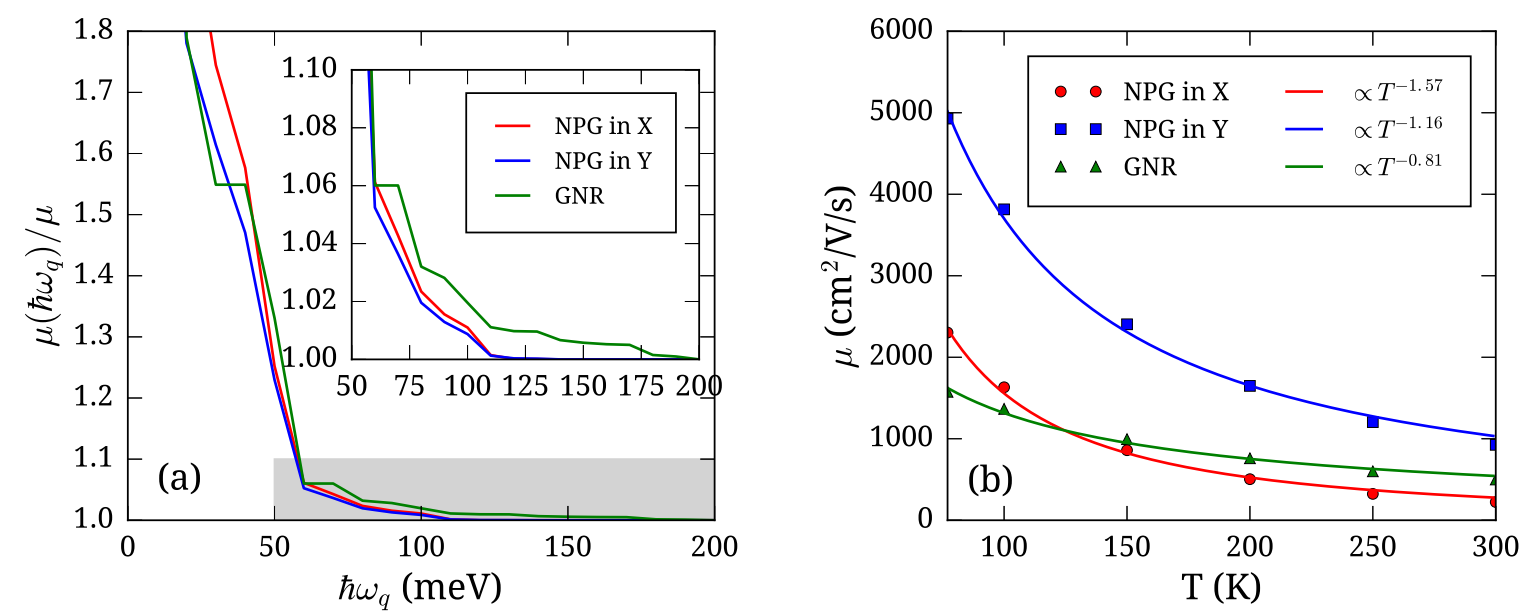

Figure 3. (a) Ratio between the mobility calculated by considering phonons with energy up to $\hbar \omega_{q}$ and the mobility calculated with all phonons, at $300 \mathrm{~K}$, for NPG and the 7-13-AGNR.(b) Electron mobility versus temperature in NPG (red disks: along X; blue squares: along Y) and in the 7-13-AGNR (green triangles). Calculations in both (a) and (b) are at low carrier density $\left(n=1.8 \times 10^{9} \mathrm{~cm}^{-2}\right)$.

characterized by electronic bands with parabolic energy dispersion. However, the energy of the phonons involved in the scattering processes extends up to $\sim 60 \mathrm{meV}$, instead of $\sim 25 \mathrm{meV}$ for semiconducting carbon nanotubes. This is due to the nanoscale patterning in NPG which tends to break the selection rules for electron-phonon scattering processes. In the 7-13-AGNR, phonon scattering is also dominated by acoustic phonons but there is a small but visible contribution from higher-energy phonons $(>60 \mathrm{meV})$ which is therefore induced by the presence of the edges.

Figure 3(b) shows that the electron mobility in NPG along the $\mathrm{Y}$ direction and in the 7-13-AGNR varies with temperature approximately as $T^{-\eta}$ with an exponent $\eta$ close to unity, as found in semiconducting carbon nanotubes [49]. Such a $1 / T$ behavior is characteristic of a 1D transport in a parabolic band, as shown using a simple analytic model (see Supplementary Material). On the contrary, the exponent close to 1.5 for the mobility in NPG along the transverse direction X may be interpreted by a 2D-like transport. This confirms the anisotropic character of the transport in NPG.

\section{Conclusion}

In conclusion, we have shown that NPG could be a good candidate for CFET applications, for beyond-CMOS technologies. NPG is characterized by a substantial energy gap, semiconducting properties, and electron-hole symmetry. The roomtemperature intrinsic carrier mobility calculated using a fully-atomistic approach is found to be strongly anisotropic and could reach high values at low carrier density, up to $800 \mathrm{~cm}^{2} /(\mathrm{Vs})$ along the main transport direction. Due to the large unit cell of the 2D material, many phonon modes are involved in the scattering processes but the 
transport is mainly limited by the coupling to acoustic phonons, approximately like in semiconducting carbon nanotubes.

\section{References}

[1] Qing Hua Wang, Kourosh Kalantar-Zadeh, Andras Kis, Jonathan N. Coleman, and Michael S. Strano. Electronics and optoelectronics of two-dimensional transition metal dichalcogenides. Nature Nanotech., 7:699, Nov 2012.

[2] Mahmut Tosun, Steven Chuang, Hui Fang, Angada B. Sachid, Mark Hettick, Yongjing Lin, Yuping Zeng, and Ali Javey. High-gain inverters based on wse2 complementary field-effect transistors. ACS Nano, 8(5):4948-4953, 2014.

[3] Saptarshi Das, Madan Dubey, and Andreas Roelofs. High gain, low noise, fully complementary logic inverter based on bi-layer wse2 field effect transistors. Appl. Phys. Lett., 105(8):083511, 2014.

[4] Ah-Jin Cho, Kee Chan Park, and Jang-Yeon Kwon. A high-performance complementary inverter based on transition metal dichalcogenide field-effect transistors. Nanoscale Res. Lett., 10(1):115, Mar 2015.

[5] Manish Chhowalla, Debdeep Jena, and Hua Zhang. Two-dimensional semiconductors for transistors. Nature Review Materials, 1:16052, 2016.

[6] Joshua A. Robinson. Perspective: 2d for beyond cmos. APL Materials, 6(5):058202, 2018.

[7] A. H. Castro Neto, F. Guinea, N. M. R. Peres, K. S. Novoselov, and A. K. Geim. The electronic properties of graphene. Rev. Mod. Phys., 81:109-162, Jan 2009.

[8] Kyoko Nakada, Mitsutaka Fujita, Gene Dresselhaus, and Mildred S. Dresselhaus. Edge state in graphene ribbons: Nanometer size effect and edge shape dependence. Phys. Rev. B, 54:1795417961, Dec 1996.

[9] Young-Woo Son, Marvin L. Cohen, and Steven G. Louie. Energy gaps in graphene nanoribbons. Phys. Rev. Lett., 97:216803, Nov 2006.

[10] Xinran Wang, Yijian Ouyang, Liying Jiao, Hailiang Wang, Liming Xie, Justin Wu, Jing Guo, and Hongjie Dai. Graphene nanoribbons with smooth edges behave as quantum wires. Nature Nanotech., 6(9):563-567, Sep 2011.

[11] Jens Baringhaus, Ming Ruan, Frederik Edler, Antonio Tejeda, Muriel Sicot, Taleb-IbrahimiAmina, An-Ping Li, Zhigang Jiang, Edward H. Conrad, Claire Berger, Christoph Tegenkamp, and Walt A. de Heer. Exceptional ballistic transport in epitaxial graphene nanoribbons. Nature, 506(7488):349-354, Feb 2014.

[12] Gábor Zsolt Magda, Xiaozhan Jin, Imre Hagymási, Péter Vancsó, Zoltán Osváth, Péter NemesIncze, Chanyong Hwang, László P. Biró, and Levente Tapasztó. Room-temperature magnetic order on zigzag edges of narrow graphene nanoribbons. Nature, 514(7524):608-611, Oct 2014.

[13] Zhiyuan Zeng, Zongyou Yin, Xiao Huang, Hai Li, Qiyuan He, Gang Lu, Freddy Boey, and Hua Zhang. Single-layer semiconducting nanosheets: High-yield preparation and device fabrication. Angew. Chem., 50(47):11093-11097, 2011.

[14] B. Radisavljevic, A. Radenovic, J. Brivio, V. Giacometti, and A. Kis. Single-layer $\operatorname{mos}_{2}$ transistors. Nature Nanotech., 6:147, Jan 2011.

[15] Sunkook Kim, Aniruddha Konar, Wan-Sik Hwang, Jong Hak Lee, Jiyoul Lee, Jaehyun Yang, Changhoon Jung, Hyoungsub Kim, Ji-Beom Yoo, Jae-Young Choi, Yong Wan Jin, Sang Yoon Lee, Debdeep Jena, Woong Choi, and Kinam Kim. High-mobility and low-power thin-film transistors based on multilayer mos2 crystals. Nat. Commun., 3:1011, Aug 2012.

[16] Hui Fang, Steven Chuang, Ting Chia Chang, Kuniharu Takei, Toshitake Takahashi, and Ali Javey. High-performance single layered wse 2 p-fets with chemically doped contacts. Nano Lett., 12(7):3788-3792, 2012.

[17] Wenxu Zhang, Zhishuo Huang, Wanli Zhang, and Yanrong Li. Two-dimensional semiconductors with possible high room temperature mobility. Nano Res., 7(12):1731-1737, 2014. 
[18] Yang Cui, Run Xin, Zhihao Yu, Yiming Pan, Zhun-Yong Ong, Xiaoxu Wei, Junzhuan Wang, Haiyan Nan, Zhenhua Ni, Yun Wu, Tangsheng Chen, Yi Shi, Baigeng Wang, Gang Zhang, Yong-Wei Zhang, and Xinran Wang. High-performance monolayer ws2 field-effect transistors on high-k dielectrics. Adv. Mater., 27(35):5230-5234, 2015.

[19] Sajedeh Manzeli, Dmitry Ovchinnikov, Diego Pasquier, Oleg V. Yazyev, and Andras Kis. 2d transition metal dichalcogenides. Nature Reviews Materials, 2, 2017.

[20] Wai Leong Chow, Peng Yu, Fucai Liu, Jinhua Hong, Xingli Wang, Qingsheng Zeng, Chuang Han Hsu, Chao Zhu, Jiadong Zhou, Xiaowei Wang, Juan Xia, Jiaxu Yan, Yu Chen, Di Wu, Ting Yu, Zexiang Shen, Hsin Lin, Chuanhong Jin, Beng Kang Tay, and Zheng Liu. High mobility 2d palladium diselenide field-effect transistors with tunable ambipolar characteristics. Adv. Mater., 29(21):1-8, 2017.

[21] Zhihao Yu, Zhun Yong Ong, Songlin Li, Jian Bin Xu, Gang Zhang, Yong Wei Zhang, Yi Shi, and Xinran Wang. Analyzing the carrier mobility in transition-metal dichalcogenide mos2field-effect transistors. Adv. Funct. Mater., 27(19), 2017.

[22] Ashima Rawat, Nityasagar Jena, Dimple, and Abir De Sarkar. A comprehensive study on carrier mobility and artificial photosynthetic properties in group vi b transition metal dichalcogenide monolayers. J. Mater. Chem. A, 6(18):8693-8704, 2018.

[23] Han Liu, Adam T. Neal, Zhen Zhu, Zhe Luo, Xianfan Xu, David Tománek, and Peide D. Ye. Phosphorene: An unexplored 2d semiconductor with a high hole mobility. ACS Nano, 8(4):40334041, 2014.

[24] Steven P. Koenig, Rostislav A. Doganov, Hennrik Schmidt, A. H. Castro Neto, and Barbaros Özyilmaz. Electric field effect in ultrathin black phosphorus. Appl. Phys. Lett., 104(10):103106, 2014.

[25] Likai Li, Yijun Yu, Guo Jun Ye, Qingqin Ge, Xuedong Ou, Hua Wu, Donglai Feng, Xian Hui Chen, and Yuanbo Zhang. Black phosphorus field-effect transistors. Nature Nanotech., 9:372, Mar 2014.

[26] Michele Buscema, Dirk J. Groenendijk, Sofya I. Blanter, Gary A. Steele, Herre S. J. van der Zant, and Andres Castellanos-Gomez. Fast and broadband photoresponse of few-layer black phosphorus field-effect transistors. Nano Lett., 14(6):3347-3352, 2014.

[27] Fengnian Xia, Han Wang, and Yichen Jia. Rediscovering black phosphorus as an anisotropic layered material for optoelectronics and electronics. Nat. Commun., 5:4458, Jul 2014.

[28] Saptarshi Das, Wei Zhang, Marcel Demarteau, Axel Hoffmann, Madan Dubey, and Andreas Roelofs. Tunable transport gap in phosphorene. Nano Lett., 14(10):5733-5739, 2014.

[29] L. Gomez, I. Aberg, and J. L. Hoyt. Electron transport in strained-silicon directly on insulator ultrathin-body n-mosfets with body thickness ranging from 2 to $25 \mathrm{~nm}$. IEEE Electron Device Lett., 28(4):285-287, April 2007.

[30] Kristen Kaasbjerg, Kristian S. Thygesen, and Karsten W. Jacobsen. Phonon-limited mobility in $n$-type single-layer $\operatorname{mos}_{2}$ from first principles. Phys. Rev. B, 85:115317, Mar 2012.

[31] Kristen Kaasbjerg, Kristian S. Thygesen, and Antti-Pekka Jauho. Acoustic phonon limited mobility in two-dimensional semiconductors: Deformation potential and piezoelectric scattering in monolayer $\operatorname{mos}_{2}$ from first principles. Phys. Rev. B, 87:235312, Jun 2013.

[32] Xiaodong Li, Jeffrey T. Mullen, Zhenghe Jin, Kostyantyn M. Borysenko, M. Buongiorno Nardelli, and Ki Wook Kim. Intrinsic electrical transport properties of monolayer silicene and $\operatorname{mos}_{2}$ from first principles. Phys. Rev. B, 87:115418, Mar 2013.

[33] Oscar D Restrepo, Kevin E Krymowski, Joshua Goldberger, and Wolfgang Windl. A first principles method to simulate electron mobilities in 2d materials. New J. Phys., 16(10):105009, 2014.

[34] Tue Gunst, Troels Markussen, Kurt Stokbro, and Mads Brandbyge. First-principles method for electron-phonon coupling and electron mobility: Applications to two-dimensional materials. Phys. Rev. B, 93(3):1-14, 2016.

[35] S. Bohloul, L. Zhang, K. Gong, and H. Guo. Theoretical impurity-limited carrier mobility of monolayer black phosphorus. Appl. Phys. Lett., 108(3), 2016. 
[36] Pere Miró, Martha Audiffred, and Thomas Heine. An atlas of two-dimensional materials. Chem. Soc. Rev., 43:6537-6554, 2014.

[37] Jingwei Bai, Xing Zhong, Shan Jiang, Yu Huang, and Xiangfeng Duan. Graphene nanomesh. Nature Nanotech., 5:190, Feb 2010.

[38] Xiaogan Liang, Yeon-Sik Jung, Shiwei Wu, Ariel Ismach, Deirdre L. Olynick, Stefano Cabrini, and Jeffrey Bokor. Formation of bandgap and subbands in graphene nanomeshes with sub-10 nm ribbon width fabricated via nanoimprint lithography. Nano Lett., 10(7):2454-2460, 2010.

[39] César Moreno, Manuel Vilas-Varela, Bernhard Kretz, Aran Garcia-Lekue, Marius V. Costache, Markos Paradinas, Mirko Panighel, Gustavo Ceballos, Sergio O. Valenzuela, Diego Peña, and Aitor Mugarza. Bottom-up synthesis of multifunctional nanoporous graphene. Science, 360(6385):199-203, 2018.

[40] Cesar Moreno, Markos Paradinas, Manuel Vilas-Varela, Mirko Panighel, Gustavo Ceballos, Diego Peña, and Aitor Mugarza. On-surface synthesis of superlattice arrays of ultra-long graphene nanoribbons. Chem. Commun., 54:9402-9405, 2018.

[41] César Moreno, Mirko Panighel, Manuel Vilas-Varela, Guillaume Sauthier, Maria Tenorio, Gustavo Ceballos, Diego Peña, and Aitor Mugarza. Critical role of phenyl substitution and catalytic substrate in the surface-assisted polymerization of dibromobianthracene derivatives. Chem. Mater., 31(2):331-341, 2019.

[42] Xiaolin Li, Xinran Wang, Li Zhang, Sangwon Lee, and Hongjie Dai. Chemically derived, ultrasmooth graphene nanoribbon semiconductors. Science, 319(5867):1229-1232, 2008.

[43] L. Wang, I. Meric, P. Y. Huang, Q. Gao, Y. Gao, H. Tran, T. Taniguchi, K. Watanabe, L. M. Campos, D. A. Muller, J. Guo, P. Kim, J. Hone, K. L. Shepard, and C. R. Dean. Onedimensional electrical contact to a two-dimensional material. Science, 342(6158):614-617, 2013.

[44] Lian-Mao Peng, Zhiyong Zhang, and Sheng Wang. Carbon nanotube electronics: recent advances. Mater. Today, 17(9):433 - 442, 2014.

[45] Zhiyong Zhang, Sheng Wang, Zhenxing Wang, Li Ding, Tian Pei, Zhudong Hu, Xuelei Liang, Qing Chen, Yan Li, and Lian-Mao Peng. Almost perfectly symmetric swcnt-based cmos devices and scaling. ACS Nano, 3(11):3781-3787, 2009.

[46] Ali Javey, Jing Guo, Damon B. Farmer, Qian Wang, Erhan Yenilmez, Roy G. Gordon, Mark Lundstrom, and Hongjie Dai. Self-aligned ballistic molecular transistors and electrically parallel nanotube arrays. Nano Lett., 4(7):1319-1322, 2004.

[47] Zhiyong Zhang, Xuelei Liang, Sheng Wang, Kun Yao, Youfan Hu, Yuzhen Zhu, Qing Chen, Weiwei Zhou, Yan Li, Yagang Yao, Jin Zhang, and Lian-Mao Peng. Doping-free fabrication of carbon nanotube based ballistic cmos devices and circuits. Nano Lett., 7(12):3603-3607, 2007.

[48] Jing Li, Y.-M. Niquet, and C. Delerue. Magnetic-phase dependence of the spin carrier mean free path in graphene nanoribbons. Phys. Rev. Lett., 116(23), 2016.

[49] Jing Li, H.P.C. Miranda, Y.-M. Niquet, L. Genovese, I. Duchemin, L. Wirtz, and C. Delerue. Phonon-limited carrier mobility and resistivity from carbon nanotubes to graphene. Phys. Rev. $B, 92(7), 2015$.

[50] Wenxing Zhang, Christophe Delerue, Yann-Michel Niquet, Guy Allan, and Enge Wang. Atomistic modeling of electron-phonon coupling and transport properties in $n$-type [110] silicon nanowires. Phys. Rev. B, 82:115319, Sep 2010.

[51] Cheol-Hwan Park, Nicola Bonini, Thibault Sohier, Georgy Samsonidze, Boris Kozinsky, Matteo Calandra, Francesco Mauri, and Nicola Marzari. Electron-phonon interactions and the intrinsic electrical resistivity of graphene. Nano Lett., 14(3):1113-1119, 2014.

[52] Thibault Sohier, Matteo Calandra, Cheol-Hwan Park, Nicola Bonini, Nicola Marzari, and Francesco Mauri. Phonon-limited resistivity of graphene by first-principles calculations: Electron-phonon interactions, strain-induced gauge field, and boltzmann equation. Phys. Rev. B, 90:125414, Sep 2014.

[53] Dmitri K. Efetov and Philip Kim. Controlling electron-phonon interactions in graphene at ultrahigh carrier densities. Phys. Rev. Lett., 105:256805, Dec 2010. 
[54] K. Zou, X. Hong, D. Keefer, and J. Zhu. Deposition of high-quality hfo2 on graphene and the effect of remote oxide phonon scattering. Phys. Rev. Lett., 105:126601, Sep 2010.

[55] Gaetano Calogero, Nick R. Papior, Bernhard Kretz, Aran Garcia-Lekue, Thomas Frederiksen, and Mads Brandbyge. Electron transport in nanoporous graphene: Probing the talbot effect. Nano Lett., 19(1):576, 2019.

[56] Jing Li, E. Lampin, C. Delerue, and Y.-M. Niquet. Theoretical investigation of the phonon-limited carrier mobility in (001) si films. J. Appl. Phys., 120(17), 2016. 


\title{
Supplementary Material for: Intrinsic transport properties of nanoporous graphene highly suitable for complementary field-effect transistors
}

\author{
Jing $\mathrm{Li}^{1, *}$ and Christophe Delerue ${ }^{2, \dagger}$ \\ ${ }^{1}$ Univ. Grenoble Alpes, CNRS, Institut NÉEL, F-38042, Grenoble, France \\ ${ }^{2}$ ULille, CNRS, Centrale Lille, ISEN, UPHF, \\ UMR 8520 - IEMN, F-59000 Lille, France
}

\begin{abstract}
A simple analytical model shows the temperature dependence of the acoustic phonon limited charge mobility for a single parabolic band is $\propto T^{-1}$ for $1 D$ system; $\propto T^{-1.5}$ for $2 D$ system; and $\propto T^{-2}$ for $3 D$ system. For an anisotropic system, the ratio of mobility between two principle axis is equal to the inverse of the ratio of effective mass, $\mu_{i i} / \mu_{j j} \propto m_{j} / m_{i}$. The analytical formula is derived with following approximations: single parabolic electron band, linear and isotropic phonon dispersion, high temperature limit, non-degenerate limit, elastic scattering approximation.
\end{abstract}

\footnotetext{
* jing.li.phy@gmail.com

† christophe.delerue@iemn.univ-lille1.fr
} 


\section{ELECTRON SCATTERING BY ACOUSTIC PHONON}

The acoustic phonon field is

$$
\hat{U}(\vec{q})=\vec{u} \sqrt{\frac{\hbar}{2 \rho V \omega_{q}}}\left(\hat{a_{q}} e^{i \vec{q} \cdot \vec{r}}+{\hat{a_{q}}}^{\dagger} e^{-i \vec{q} \cdot \vec{r}}\right)
$$

where $\vec{q}$ is the phonon wavevector, $\omega_{q}$ the phonon frequency, $\hat{a_{q}}$ and ${\hat{a_{q}}}^{\dagger}$ the creation and annihilation operators, $\rho$ the material density, $V$ the volume of the unit cell, and $\vec{u}$ the polarization unit vector of phonon.

The phonon field creates a strain field, which is

$$
\nabla \cdot \hat{U}(\vec{q})=i[\vec{q} \cdot \vec{u}] \sqrt{\frac{\hbar}{2 \rho V \omega_{q}}}\left(\hat{a_{q}} e^{i \vec{q} \cdot \vec{r}}-{\hat{a_{q}}}^{\dagger} e^{-i \vec{q} \cdot \vec{r}}\right)
$$

For simplicity, we consider a single band in electronic structure, the strain field perturbs the electron Hamiltonian through the deformation potential $D$. In this case, only the longitudinal acoustic phonon contributes, $\vec{q} \cdot \vec{u}_{L A}=q$. The transverse phonon has no contribution $\vec{q} \cdot \vec{u}_{T A}=0$. So the perturbation is

$$
\Delta H=D \nabla \cdot \hat{U}(\vec{q})=i q D \sqrt{\frac{\hbar}{2 \rho V \omega_{q}}}\left(\hat{a_{q}} e^{i q r}-\hat{a_{q}^{\dagger}} e^{-i q r}\right)
$$

Consider the initial and final electronic states, $\left|\psi_{i}\right\rangle$ and $\left|\psi_{f}\right\rangle$. The scattering rate is given by Fermi's golden rule:

$$
W_{i, f}=\frac{2 \pi}{\hbar}\left|\left\langle\psi_{i}|\Delta H| \psi_{f}\right\rangle\right|^{2} \rho(\vec{q})
$$

where the matrix element is

$$
\begin{aligned}
\left|\left\langle\psi_{i}|\Delta H| \psi_{f}\right\rangle\right|^{2}= & \frac{\hbar D^{2} q^{2}}{2 \rho V \omega_{q}}\left(\hat{a_{q}}{\hat{a_{q}}}^{\dagger}+{\hat{a_{q}}}^{\dagger} \hat{a_{q}}\right)\left|\left\langle\psi_{i}\left|e^{i \vec{q} \cdot \vec{r}}\right| \psi_{f}\right\rangle\right|^{2} \\
& =\frac{\hbar D^{2} q^{2}}{2 \rho V \omega_{q}} \operatorname{coth}\left(\frac{\hbar \omega_{q}}{2 k_{B} T}\right)\left|\left\langle\psi_{i}\left|e^{i \vec{q} \cdot \vec{r}}\right| \psi_{f}\right\rangle\right|^{2}
\end{aligned}
$$

The scattering density $\rho(\vec{q})$ depends on the dimensionality of the system

$$
\rho_{1 D}(q)=\frac{L}{2 \pi \hbar v_{p h}} ; \quad \rho_{2 D}(q)=\frac{S}{(2 \pi)^{2} \hbar v_{p h}} ; \quad \rho_{3 D}(q)=\frac{V}{(2 \pi)^{3} \hbar v_{p h}},
$$

where $v_{p h}$ is the phonon velocity, $L$ the length of the 1D unit cell, and $S$ the area of the 2D unit cell. 
Consider linear phonon dispersion $\omega_{q}=v_{p h} q$, at high temperature limit $k_{B} T>>\hbar \omega_{q}$, (so $\left.\operatorname{coth}\left(\frac{\hbar \omega_{q}}{2 k_{B} T}\right) \approx \frac{2 k_{B} T}{\hbar \omega_{q}},\right)$ therefore

$$
W_{i, f}=\tilde{W}\left|\left\langle\psi_{i}\left|e^{i \vec{q} \cdot \vec{r}}\right| \psi_{f}\right\rangle\right|^{2}
$$

where $\tilde{W}$ is the electronic wave-function independent scattering rate,

$$
\tilde{W}_{1 D}=\frac{L D^{2} k_{B} T}{\hbar^{2} \rho V v_{p h}^{3}} ; \quad \tilde{W}_{2 D}=\frac{S D^{2} k_{B} T}{2 \pi \hbar^{2} \rho V v_{p h}^{3}} ; \quad \tilde{W}_{3 D}=\frac{D^{2} k_{B} T}{4 \pi^{2} \hbar^{2} \rho v_{p h}^{3}} .
$$

\section{A. The mobility in $1 \mathrm{D}$ system}

Assume the scattering by acoustic phonon is elastic, for the initial state with the electron wavevector $k$, the final state is $k^{\prime}$. The Boltzmann transport equation for the initial state is written as:

$$
\sum_{k^{\prime}}\left\{f(k) W_{k, k^{\prime}}\left[1-f\left(k^{\prime}\right)\right]-[1-f(k)] W_{k^{\prime}, k} f\left(k^{\prime}\right)\right\}=\left.e F \cdot v(k)\left(\frac{\partial f^{0}}{\partial E}\right)\right|_{E(k)},
$$

where $v(k)$ is the electron group velocity, $F$ is the external electric field, $f(k)$ the occupation factor at state $k$, under the first order approximation,

$$
f(k)=f^{0}(k)+e F g(k)
$$

The term $f^{0}(k)$ is the equilibrium distribution function $f^{0}(k)=\left(1+e^{\frac{E_{k}-E_{f}}{k_{B} T}}\right)^{-1}, E_{f}$ the Fermilevel, and $T$ the temperature. Because $E(k)=E\left(k^{\prime}\right), f^{0}(k)=f^{0}\left(k^{\prime}\right)$. With $W_{k, k^{\prime}}=W_{k^{\prime}, k}$, the Eq.9 is simplified to

$$
\sum_{k^{\prime}}\left[g(k)-g\left(k^{\prime}\right)\right] W_{k, k^{\prime}}=\left.v(k)\left(\frac{\partial f^{0}}{\partial E}\right)\right|_{E_{k}},
$$

by keeping only the first order term in distribution function to the external electric field. For the reason of the charge conservation, $\sum_{k} g(k)=0$. So, we have

$$
g(k)=\left.\frac{1}{W_{k,-k}} v(k)\left(\frac{\partial f^{0}}{\partial E}\right)\right|_{E_{k}}
$$

The mobility is given by

$$
\mu=-e \frac{\int g(k) v(k) d k}{\int f^{0}(k) d k}
$$

Consider a parabolic band structure: $E(k)=\frac{\hbar^{2} k^{2}}{2 m^{*}}$, where $m^{*}$ is the effective mass, the group velocity $v(k)=\frac{\hbar k}{m^{*}}$. At non-degenerate limit $E_{k}-E_{f}>>k_{B} T, f^{0}(k) \approx e^{-\frac{E_{k}-E_{f}}{k_{B} T}}$. So

$$
g(k)=-\frac{1}{W_{k, k^{\prime}}} \frac{\hbar k}{m^{*} k_{B} T} e^{-\frac{E_{k}-E_{f}}{k_{B} T}}
$$


and the mobility is

$$
\mu^{1 D}=\frac{e \hbar^{2}}{\left(m^{*}\right)^{2} k_{B} T} \frac{\int_{0}^{\infty} \frac{k^{2}}{W_{k, k^{\prime}}} e^{-\frac{E_{k}-E_{f}}{k_{B} T}} d k}{\int_{0}^{\infty} e^{-\frac{E_{k}-E_{f}}{k_{B} T}} d k} .
$$

Assume $\left|\left\langle\psi_{i}\left|e^{i q r}\right| \psi_{f}\right\rangle\right|^{2}=I$ is a constant for all scattering events, $W_{k, k^{\prime}}=\tilde{W}_{1 D} I$ is energy independent.

$$
\mu^{1 D}=\frac{e \hbar^{2}}{\left(m^{*}\right)^{2} \tilde{W}_{1 D} I k_{B} T} \frac{\int_{0}^{\infty} k^{2} e^{-\frac{E_{k}-E_{f}}{k_{B} T}} d k}{\int_{0}^{\infty} e^{-\frac{E_{k}-E_{f}}{k_{B} T}} d k},
$$

After the integrals,

$$
\begin{array}{r}
\int_{0}^{\infty} k^{2} e^{-\frac{E_{k}-E_{f}}{k_{B} T}} d k=\sqrt{\frac{\pi}{2}} \frac{\left(m^{*} k_{B} T\right)^{\frac{3}{2}}}{\hbar^{3}} e^{\frac{E_{f}}{k_{B} T}} \\
\int_{0}^{\infty} e^{-\frac{E_{k}-E_{f}}{k_{B} T}} d k=\frac{\sqrt{\pi m^{*} k_{B} T}}{\sqrt{2} \hbar} e^{\frac{E_{f}}{k_{B} T}}
\end{array}
$$

the mobility is then

$$
\mu^{1 D}=\frac{e}{m^{*} \tilde{W}_{1 D} I}=\frac{e \hbar^{2} \rho V v_{p h}^{3}}{L D^{2} I k_{B} T m^{*}},
$$

which is $\propto T^{-1}$.

\section{B. The mobility in $2 \mathrm{D}$ system}

Assume the scattering by acoustic phonon is elastic, for the initial state with the electron wavevector $k$, the final states $k^{\prime}$ are on the equal energy circle or ellipse, $E_{k}^{\prime}=E_{k}$. The Boltzmann transport equation for the initial state is written as:

$$
\int f(k) W_{k, k^{\prime}}\left[1-f\left(k^{\prime}\right)\right]-[1-f(k)] W_{k^{\prime}, k} f\left(k^{\prime}\right) d k^{\prime}=\left.e F \cdot v(k)\left(\frac{\partial f^{0}}{\partial E}\right)\right|_{E(k)} .
$$

Similar to the $1 \mathrm{D}$ case, with $W_{k, k^{\prime}}=W_{k^{\prime}, k}$, the equation is is simplified to

$$
\int\left[g(k)-g\left(k^{\prime}\right)\right] W_{k, k^{\prime}} d k^{\prime}=\left.\hat{F} \cdot v(k)\left(\frac{\partial f^{0}}{\partial E}\right)\right|_{E_{k}}
$$

by keeping only the first order term in distribution function to the external electric field, whose unit vector is $\hat{F}$. Assume $\left|\left\langle\psi_{i}\left|e^{i q r}\right| \psi_{f}\right\rangle\right|^{2}=I$ is a constant for all scattering events, $W_{k,-k}=\tilde{W}_{2 D} I$ is independent of $k$ and $k^{\prime}$. With the conservation of charge $\int g(k) d k=0$, the equation is further simplified to

$$
C_{k} g(k) \tilde{W}_{2 D} I=\left.\hat{F} \cdot v(k)\left(\frac{\partial f^{0}}{\partial E}\right)\right|_{E_{k}},
$$


where $C_{k}$ is the circumference of the equal energy circle or ellipse. Hence,

$$
g(k)=\left.\frac{1}{C_{k} \tilde{W}_{2 D} I} \hat{F} \cdot v(k)\left(\frac{\partial f^{0}}{\partial E}\right)\right|_{E_{k}} .
$$

Consider the electronic structure, $E(k)=\frac{\hbar k_{x}^{2}}{2 m_{x}}+\frac{\hbar k_{y}^{2}}{2 m_{y}}$, where $m_{x}$ and $m_{y}$ are the effective mass in $\hat{x}$ and $\hat{y}$. For the convenient of integral, let's introduce $\tilde{k_{x}}=\frac{k_{x}}{\sqrt{m_{x}}}$ and $\tilde{k_{y}}=\frac{k_{y}}{\sqrt{m_{y}}}$, so $E(k)=\frac{\hbar}{2} \tilde{k}^{2}$, with $\tilde{k}^{2}=\tilde{k}_{x}^{2}+\tilde{k}_{y}^{2}$. The group velocity is $\vec{v}(\tilde{k})=\frac{\hbar \tilde{k}_{x}}{\sqrt{m_{x}}} \hat{x}+\frac{\hbar \tilde{k}_{y}}{\sqrt{m_{y}}} \hat{y}$. The circumference $C_{k}$ is approximated to

$$
C_{k} \approx 2 \pi \sqrt{\frac{a^{2}+b^{2}}{2}}=2 \pi \tilde{k} \sqrt{\frac{m_{x}+m_{y}}{2}}
$$

where $a=\sqrt{m_{x}} \tilde{k}$ and $b=\sqrt{m_{y}} \tilde{k}$ are the principle axis of the equal energy ellipse. At non-degenerate limit $E_{k}-E_{f}>>k_{B} T, f^{0}(k) \approx e^{-\frac{E_{k}-E_{f}}{k_{B} T}}$, the mobility (Eq.13) is

$$
\mu^{2 D}=\frac{e}{\tilde{W}_{2 D} I k_{B} T} \frac{\int_{0}^{\infty} \int_{0}^{2 \pi} \frac{[\hat{F} \cdot v(k)] v(k)}{C_{k}} e^{-\frac{E_{k}-E_{f}}{k_{B} T}} \tilde{k} d \theta d \tilde{k}}{\int_{0}^{\infty} \int_{0}^{2 \pi} e^{-\frac{E_{k}-E_{f}}{k_{B} T}} \tilde{k} d \theta d \tilde{k}}
$$

The integral in the denominator is

$$
\int_{0}^{\infty} \int_{0}^{2 \pi} e^{-\frac{E_{k}-E_{f}}{k_{B} T}} \tilde{k} d \theta d \tilde{k}=\frac{2 \pi k_{B} T}{\hbar^{2}} e^{\frac{E_{f}}{k_{B} T}}
$$

The integral at the numerator contains the velocity, which is a vector, we can evaluate their component separately. For external field in $\hat{x}$, consider velocity in $\hat{x}$, we have:

$$
\int_{0}^{\infty} \int_{0}^{2 \pi} \frac{v(k)_{x}^{2}}{C_{k}} e^{-\frac{E_{k}-E_{f}}{k_{B} T}} \tilde{k} d \theta d \tilde{k}=\frac{\sqrt{\pi}\left(k_{B} T\right)^{3 / 2}}{2 \hbar m_{x} \sqrt{m_{x}+m_{y}}} e^{\frac{E_{f}}{k_{B} T}}
$$

For external field in $\hat{y}$ and velocity in $\hat{y}$, the integral is

$$
\int_{0}^{\infty} \int_{0}^{2 \pi} \frac{v(k)_{y}^{2}}{C_{k}} e^{-\frac{E_{k}-E_{f}}{k_{B} T}} \tilde{k} d \theta d \tilde{k}=\frac{\sqrt{\pi}\left(k_{B} T\right)^{3 / 2}}{2 \hbar m_{y} \sqrt{m_{x}+m_{y}}} e^{\frac{E_{f}}{k_{B} T}}
$$

The integral is zero if the velocity component is perpendicular to the external field. The mobility is then

$$
\begin{aligned}
\mu_{x x}^{2 D} & =\frac{e \pi^{1 / 2} \hbar^{3} \rho V v_{p h}^{3}}{2 S D^{2} I\left(k_{B} T\right)^{3 / 2}\left(m_{x}+m_{y}\right)^{1 / 2} m_{x}} ; \\
\mu_{y y}^{2 D} & =\frac{e \pi^{1 / 2} \hbar^{3} \rho V v_{p h}^{3}}{2 S D^{2} I\left(k_{B} T\right)^{3 / 2}\left(m_{x}+m_{y}\right)^{1 / 2} m_{y}} ;
\end{aligned}
$$

which are $\propto T^{-1.5}$, and $\mu_{x x} / \mu_{y y}=m_{y} / m_{x}$. 


\section{The mobility in $3 \mathrm{D}$ system}

Assume the scattering by acoustic phonon is elastic, for the initial state with the electron wavevector $k$, the final states $k^{\prime}$ are on the equal energy spheroid, $E_{k}^{\prime}=E_{k}$. The Boltzmann transport equation for the initial state is written as:

$$
\int f(k) W_{k, k^{\prime}}\left[1-f\left(k^{\prime}\right)\right]-[1-f(k)] W_{k^{\prime}, k} f\left(k^{\prime}\right) d k^{\prime}=\left.e F \cdot v(k)\left(\frac{\partial f^{0}}{\partial E}\right)\right|_{E(k)} .
$$

Analogy to the $2 \mathrm{D}$ case, with $W_{k, k^{\prime}}=W_{k^{\prime}, k}$, the equation is is simplified to

$$
\int\left[g(k)-g\left(k^{\prime}\right)\right] W_{k, k^{\prime}} d k^{\prime}=\left.\hat{F} \cdot v(k)\left(\frac{\partial f^{0}}{\partial E}\right)\right|_{E_{k}},
$$

by keeping only the first order term in distribution function to the external electric field, whose unit vector is $\hat{F}$. Assume $\left|\left\langle\psi_{i}\left|e^{i q r}\right| \psi_{f}\right\rangle\right|^{2}=I$ is a constant for all scattering events, $W_{k,-k}=\tilde{W}_{3 D} I$ is independent of $k$ and $k^{\prime}$. With the conservation of charge $\int g(k) d k=0$, the equation is further simplified to

$$
S_{k} g(k) \tilde{W}_{3 D} I=\left.\hat{F} \cdot v(k)\left(\frac{\partial f^{0}}{\partial E}\right)\right|_{E_{k}},
$$

where $S_{k}$ is the surface of the equal energy spheroid. Hence,

$$
g(k)=\left.\frac{1}{S_{k} \tilde{W}_{3 D} I} \hat{F} \cdot v(k)\left(\frac{\partial f^{0}}{\partial E}\right)\right|_{E_{k}} .
$$

Consider the electronic structure, $E(k)=\frac{\hbar k_{x}^{2}}{2 m_{x}}+\frac{\hbar k_{y}^{2}}{2 m_{y}}+\frac{\hbar k_{z}^{2}}{2 m_{z}}$, where $m_{x}, m_{y}$, and $m_{z}$ are the effective mass in $\hat{x}, \hat{y}$, and $\hat{z}$. For the convenient of integral, let's introduce $\tilde{k_{x}}=\frac{k_{x}}{\sqrt{m_{x}}}$, $\tilde{k_{y}}=\frac{k_{y}}{\sqrt{m_{y}}}$, and $\tilde{k}_{z}=\frac{k_{z}}{\sqrt{m_{z}}}$, so $E(k)=\frac{\hbar}{2} \tilde{k}^{2}$, with $\tilde{k}^{2}=\tilde{k}_{x}^{2}+\tilde{k}_{y}^{2}+\tilde{k}_{z}^{2}$. The group velocity is $\vec{v}(\tilde{k})=\frac{\hbar \tilde{k}_{x}}{\sqrt{m_{x}}} \hat{x}+\frac{\hbar \tilde{k}_{y}}{\sqrt{m_{y}}} \hat{y}+\frac{\hbar \tilde{k}_{z}}{\sqrt{m_{z}}} \hat{z}$. The surface of the spheroid $S_{k}$ is approximated to

$$
S_{k} \approx 2 \pi \sqrt{a^{2} b^{2}+a^{2} c^{2}+b^{2} c^{2}}=2 \pi \tilde{k}^{2} \sqrt{m_{x} m_{y}+m_{x} m_{z}+m_{y} m_{z}}
$$

where $a=\sqrt{m_{x}} \tilde{k}, b=\sqrt{m_{y}} \tilde{k}$, and $c=\sqrt{m_{z}} \tilde{k}$ are the principle axis of the equal energy spheroid. At non-degenerate limit $E_{k}-E_{f}>>k_{B} T, f^{0}(k) \approx e^{-\frac{E_{k}-E_{f}}{k_{B} T}}$, the mobility (Eq.13) is

$$
\mu^{3 D}=\frac{e}{\tilde{W}_{3 D} I k_{B} T} \frac{\int_{0}^{\infty} \int_{0}^{\pi} \int_{0}^{2 \pi} \frac{[\hat{F} \cdot v(k)] v(k)}{S_{k}} e^{-\frac{E_{k}-E_{f}}{k_{B} T}} \tilde{k}^{2} \sin \theta d \phi d \theta d \tilde{k}}{\int_{0}^{\infty} \int_{0}^{\pi} \int_{0}^{2 \pi} e^{-\frac{E_{k}-E_{f}}{k_{B} T}} \tilde{k}^{2} \sin \theta d \phi d \theta d \tilde{k}} .
$$

The integral in the denominator is

$$
\int_{0}^{\infty} \int_{0}^{\pi} \int_{0}^{2 \pi} e^{-\frac{E_{k}-E_{f}}{k_{B} T}} \tilde{k}^{2} \sin \theta d \phi d \theta d \tilde{k}=\frac{\left(2 \pi k_{B} T\right)^{3 / 2}}{\hbar^{3}} e^{\frac{E_{f}}{k_{B} T}} .
$$


The integral at the numerator contains the velocity, which is a vector, we can evaluate their component separately. For external field in $\hat{x}$, consider velocity in $\hat{x}$, we have:

$$
\int_{0}^{\infty} \int_{0}^{\pi} \int_{0}^{2 \pi} \frac{v(k)_{x}^{2}}{S_{k}} e^{-\frac{E_{k}-E_{f}}{k_{B} T}} \tilde{k}^{2} \sin \theta d \phi d \theta d \tilde{k}=\frac{\sqrt{2 \pi}\left(k_{B} T\right)^{3 / 2}}{3 \hbar m_{x} \sqrt{m_{x} m_{y}+m_{x} m_{z}+m_{y} m_{z}}} e^{\frac{E_{f}}{k_{B} T}}
$$

For external field in $\hat{y}$ and velocity in $\hat{y}$, the integral is

$$
\int_{0}^{\infty} \int_{0}^{\pi} \int_{0}^{2 \pi} \frac{v(k)_{y}^{2}}{S_{k}} e^{-\frac{E_{k}-E_{f}}{k_{B} T}} \tilde{k}^{2} \sin \theta d \phi d \theta d \tilde{k}=\frac{\sqrt{2 \pi}\left(k_{B} T\right)^{3 / 2}}{3 \hbar m_{y} \sqrt{m_{x} m_{y}+m_{x} m_{z}+m_{y} m_{z}}} e^{\frac{E_{f}}{k_{B} T}} .
$$

, and for external field in $\hat{z}$ and velocity in $\hat{z}$, the integral is

$$
\int_{0}^{\infty} \int_{0}^{\pi} \int_{0}^{2 \pi} \frac{v(k)_{z}^{2}}{S_{k}} e^{-\frac{E_{k}-E_{f}}{k_{B} T}} \tilde{k}^{2} \sin \theta d \phi d \theta d \tilde{k}=\frac{\sqrt{2 \pi}\left(k_{B} T\right)^{3 / 2}}{3 \hbar m_{z} \sqrt{m_{x} m_{y}+m_{x} m_{z}+m_{y} m_{z}}} e^{\frac{E_{f}}{k_{B} T}} .
$$

The integral is zero if the velocity component is perpendicular to the external field. The mobility is then

$$
\begin{aligned}
\mu_{x x}^{3 D} & =\frac{2 e \pi^{3 / 2} \hbar^{4} \rho v_{p h}^{3}}{3 D^{2} I\left(k_{B} T\right)^{2}\left(m_{x} m_{y}+m_{x} m_{z}+m_{y} m_{z}\right)^{1 / 2} m_{x}} ; \\
\mu_{y y}^{3 D} & =\frac{2 e \pi^{3 / 2} \hbar^{4} \rho v_{p h}^{3}}{3 D^{2} I\left(k_{B} T\right)^{2}\left(m_{x} m_{y}+m_{x} m_{z}+m_{y} m_{z}\right)^{1 / 2} m_{y}} ; \\
\mu_{z z}^{3 D} & =\frac{2 e \pi^{3 / 2} \hbar^{4} \rho v_{p h}^{3}}{3 D^{2} I\left(k_{B} T\right)^{2}\left(m_{x} m_{y}+m_{x} m_{z}+m_{y} m_{z}\right)^{1 / 2} m_{z}}
\end{aligned}
$$

which are $\propto T^{-2}$, and the ratio of mobility between two principle axis: $\mu_{i i} / \mu_{j j}=m_{j} / m_{i}$, $i, j \in[x, y, z]$. 